\title{
Biotechnology Methods
}

E. Heinzle

Mass Spectrometry for On-line Monitoring of Biotechnological Processes

C. Bedetti, A. Cantafora

Extraction and Purification of Arachidonic Acid Metabolites from Cell Cultures

Ch. S. Ho, M. D. Smith, J. F. Shanahan

Carbon Dioxide Transfer in Biochemical Reactors

M. J. Beker, A. I. Rapoport

Conservation of Yeasts by Dehydration

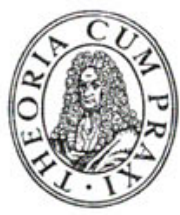



Biotechnology Methods 



\section{Biotechnology Methods}

Managing Editor: A. Fiechter

With 71 Figures and 29 Tables

Akademie-Verlag Berlin 1988 
Die Originalausgabe erscheint im Springer-Verlag Berlin Heidelberg New York als Volume 35 der Schriftenreihe

Advances in Biochemical Engineering/Biotechnology

Vertrieb ausschließlich für die DDR und die sozialistischen Länder Akademie-Verlag Berlin

Alle Rechte vorbehalten

(C) Springer-Verlag Berlin Heidelberg 1987

ISBN 3-540-17627-6 Springer-Verlag Berlin Heidelberg New York Tokyo

ISBN 0-387-17627-6 Springer-Verlag New York Heidelberg Berlin Tokyo

ISBN 3-05-500506-6

Erschienen im Akademie-Verlag Berlin, DDR-1086 Berlin, Leipziger Straße 3-4

Lizenznummer: $202 \cdot 100 / 523 / 87$

Printed in the German Democratic Republic

Gesamtherstellung: VEB Druckerei „Thomas Müntzer“, 5820 Bad Langensalza

LSV 1315

Bestellnummer: $7637586(3070 / 35)$

12800 


\section{Managing Editor}

Professor Dr. A. Fiechter

Institut für Biotechnologie, Eidgenössische Technische Hochschule ETH - Hönggerberg, CH-8093 Zürich

\section{Editorial Board}

Prof. Dr. S. Aiba

Prof. Dr. H. R. Bungay

Prof. Dr. Ch. L. Cooney

Prof. Dr. A. L. Demain

Prof. Dr. S. Fukui

Prof. Dr. K. Kieslich

Prof. Dr. A. M. Klibanov

Prof. Dr. R. M. Lafferty

Prof. Dr. B. S. Montenecourt

Prof. Dr. S. B. Primrose

Prof. Dr. H. J. Rehm

Prof. Dr. P. L. Rogers

Prof. Dr. H. Sahm

Prof. Dr. K. Schügerl

Prof. Dr. S. Suzuki

Prof. Dr. H. Taguchi

Prof. Dr. G. T. Tsao

Dr. K. Venkat

Prof. Dr. E.-L. Winnacker
Department of Fermentation Technology, Faculty of Engineering, Osaka University, Yamada-Kami, SuitaShi, Osaka 565, Japan

Rensselaer Polytechnic Institute, Dept. of Chem. and Environment. Engineering, Troy, NY 12180-3590/USA

Massachusetts Institute of Technology,

Department of Chemical Engineering, Cambridge, Massachusetts 02139/USA

Massachusetts Institute of Technology, Dept. of

Nutrition \& Food Sc., Room 56-125

Cambridge, Massachusetts 02139/USA

Dept. of Industrial Chemistry, Faculty of Engineering, Sakyo-Ku, Kyoto 606, Japan

Gesellschaft für Biotechnologie, Forschung $\mathrm{mbH}$, Mascheroder Weg 1, D-3300 Braunschweig

Massachusetts Institute of Technology, Dept. of Applied Biological Sciences, Cambridge, Massachusetts 02139/ USA

Techn. Hochschule Graz, Institut für Biochem. Technol., Schlögelgasse 9, A-8010 Graz

Lehigh University, Biolog. and Biotechnology Research Center, Bethlehem, PA 18015/USA

General Manager, Molecular Biology Division, Amersham International plc., White Lion Road Amersham, Buckinghamshire HP7 9LL, England

Westf. Wilhelms Universität, Institut für Mikrobiologie, Corrensstr. 3, D-4400 Münster

School of Biological Technology, The University of New South Wales. P.O. Box 1,

Kensington, New South Wales, Australia 2033

Institut für Biotechnologie, Kernforschungsanlage Jülich, D-5170 Jülich

Institut für Technische Chemie, Universität Hannover, Callinstraße 3, D-3000 Hannover

Tokyo Institute of Technology, Nagatsuta Campus, Res. Lab. of Resources Utilization, 4259, Nagatsuta, Midori-ku, Yokohama 227/Japan

Faculty of Engineering, Osaka University, Yamada-kami, Suita-shi, Osaka 565/Japan

Director, Lab. of Renewable Resources Eng., A. A. Potter Eng. Center, Purdue University, West Lafayette, IN 47907/USA

Corporate Director Science and Technology, H. J. Heinz Company U.S. Steel Building, P.O. Box 57, Pittsburgh, PA 15230/USA

Universität München, Institut f. Biochemie, Karlsstr. 23, D-8000 München 2 
